\title{
Establishment of a microplate assay for flow cytometric assessment and it is use for the evaluation of age-related phenotypic changes in canine whole blood leukocytes
}

\author{
Alexandre B. Reis ${ }^{\mathrm{b}, \mathrm{c}, \mathrm{d}, *}$, Cláudia M. Carneiro ${ }^{\mathrm{c}, \mathrm{d}}$, Maria das Graças Carvalho ${ }^{\mathrm{e}}$, \\ Andréa Teixeira-Carvalho ${ }^{\mathrm{b}, \mathrm{c}}$, Rodolfo C. Giunchetti ${ }^{\mathrm{a}}$, Wilson Mayrink ${ }^{\mathrm{a}}$, \\ Odair Genaro $^{\mathrm{a}, 1}$, Rodrigo Corrêa-Oliveira ${ }^{\mathrm{b}}$, Olindo A. Martins-Filho ${ }^{\mathrm{f}}$ \\ a Laboratório de Leishmanioses, Departamento de Parasitologia, Instituto de Ciências Biológicas, \\ Universidade Federal de Minas Gerais, Belo Horizonte, Minas Gerais, Brazil \\ b Laboratório de Imunologia Celular e Molecular, Centro de Pesquisas René Rachou, Fundação Oswaldo Cruz, \\ Av. Augusto de Lima, 1715 Barro Preto, Belo Horizonte, MG CEP 30190-002, Minas Gerais, Brazil \\ ${ }^{\mathrm{c}}$ Departamento de Análises Clínicas, Escola de Farmácia, Universidade Federal de Ouro Preto, Ouro Preto, Minas Gerais, Brazil \\ ${ }^{\mathrm{d}}$ Laboratório de Parasitologia e Histopatologia, Núcleo de Pesquisas em Ciências Biológicas/NUPEB, \\ Instituto de Ciências Exatas e Biológicas, Universidade Federal de Ouro Preto, Ouro Preto, Minas Gerais, Brazil \\ ${ }^{\mathrm{e}}$ Laboratório de Hematologia Clínica, Faculdade de Farmácia, Universidade Federal de Minas Gerais, \\ Belo Horizonte, Minas Gerais, Brazil \\ ${ }^{\mathrm{f}}$ Laboratório de Doença de Chagas, Centro de Pesquisas René Rachou, Fundação Oswaldo Cruz, \\ Belo Horizonte, Minas Gerais, Brazil
}

Received 30 March 2004; received in revised form 23 August 2004; accepted 30 August 2004

\begin{abstract}
The effectiveness of flow cytometric assays for canine use is still requiring standardization. Despite several studies using purified mononuclear cells, no methodology or reference ranges are available for immunophenotyping of whole blood leukocytes (WBL). Fresh and pre-fixed WBL were used to identify cell-subsets, (Thy $-1^{+} / \mathrm{CD}^{+} / \mathrm{CD} 4^{+} / \mathrm{CD} 8^{+} / \mathrm{CD} 21^{+}$and $\mathrm{CD}_{1} 4^{+}$) and measure MHC-II, CD45RA/CD45RB expression. We described here an efficient method for fast quantification of canine-WBL, using pre-fix in a microplate assay, which allows long-term sample storage prior to phenotyping. Decreased percentage of $\mathrm{CD}^{+}$-T-cells within the lymphocyte-gate and increased percentage of $\mathrm{CD} 21^{+}$-B-cells were observed in young animals, which led to higher T/B cell ratios in middle-aged dogs. Lower numerical counts of Thy- $1^{+}, \mathrm{CD} 4^{+}, \mathrm{CD} 8^{+}$and $\mathrm{CD} 21^{+}$ lymphocyte were observed when compared to young animals. In addition, we identified an age-related decline of MHC-II/ CD45RA expression by lymphocytes. We proposed an improved method for phenotyping of canine peripheral blood mononuclear cells (PBMC) that has significant use for researchers and veterinary clinicians. The hematological changes of senescence previously identified on PBMC could be adequately reproduced on features identified by whole blood. Furthermore,
\end{abstract}

\footnotetext{
* Corresponding author. Tel.: +55 313295 3566; fax: +55 3132953115.

E-mail address: alexreis@cpqrr.fiocruz.br (A.B. Reis).

${ }^{1}$ In Memoriam.
} 
this study supplies normal range references as baseline standards for clinical purposes, besides specific immunological parameters to monitor canine aging process.

(C) 2004 Elsevier B.V. All rights reserved.

Keywords: Flow cytometry; Canine; Peripheral blood; Lymphocyte subsets; Aging and sex

\section{Introduction}

The dog has been used as an important model to investigate both experimental and natural veterinary disorders such as autoimmune or immune mediated diseases (Williams, 1997; Pedersen and Pool, 1978). Considering the pathological similarities between dogs and humans in many diseases caused by infectious agents, several studies have utilized these animals as an alternative model to evaluate parasitehost interactions. Moreover, recent advances in the immunology require improvement of the methodology to be applied in clinical and experimental systems. Therefore, significant effort is being devoted to the development of new methods focusing on the diagnosis, prognosis and the evaluation of therapeutic and vaccine protocols for use in routine clinical veterinary (Williams, 1997; Cobbold and Metcalfe, 1994; Pinelli, 1997).

Flow cytometry is a well-established methodology in clinical laboratories to identify cell populations and subpopulations in a broad variety of biological fluids. A large number of flow applications are becoming available due an increase in our ability to simultaneously measure distinct cell phenotypes, such as morphological features, as well as fluorescence-based protein profile. Lymphocyte phenotyping is a common application of flow cytometry and has been used to analyze the impact of diseases on the immune system, through alterations on the leukocytes of interest in clinical medicine (Jaroszeski and Radcliff, 1999).

Flow cytometry has become an even more promising tool to characterize a variety of cell subsets in several animal species, with a great interest and emphasis on studies in canine clinical veterinary. However, the effectiveness of flow cytometric assays is still requiring standardization, especially in clinical settings. Over the past few years, the major challenge has been the lack of specific reagents to investigate canine cell biology. The Canine Leukocyte Antigen
Workshop (CLAW, 1994) was a key event to encourage the scientific community to search for new flow cytometric approaches to identify canine cell subpopulations. However, to this date, despite several studies using purified canine PBMC (Byrne et al., 2000), hardly any methodology or reference ranges for lymphocyte subsets in whole blood have been published or made available to the scientific community (Otani et al., 2002).

In this study, our major goal was to develop and standardize a methodological process, named pre-fix, for canine leukocyte phenotyping, using erythrocyte previously lysed and leukocyte fixed whole blood samples. Considering the molecular stability of canine cell surface markers after fixation with formaldehyde, the pre-fix protocol brings great advantages to cell phenotyping, increasing the perspective of applying this methodology to field studies, where shipment facilities may not be available and storage could interfere on the phenotyping results. The results reported here also contribute to improvement of our ability to run a fast and efficient analysis of canine WBL subsets, applicable for the monitoring of several disorders, including onco-hematological syndromes, autoimmune and infectious diseases. As a major gain, our data supplies normal ranges references for young and middle-aged mongrel dogs, as baseline standards for clinical purposes and use this methodology on a study related to aging of dogs.

\section{Materials and methods}

\subsection{Animals}

Forty mongrel dogs, 20 males and 20 females, age ranging from 4 months (young, $n=20$ ) to 6 years (middle-aged, $n=20$ ) were maintained in quarantine, confined in the kennel at the Institute of Biological Sciences, Federal University of Minas Gerais (UFMG), Brazil. Prior to the inclusion in this study, 
all animals were treated for intestinal helminthic infections and immunized against parvovirosis, leptospirosis, distemper, parainfluenza and hepatitis. All animals received drinking water and a balanced feed (Kinus $^{\circledR}$-BRASWEY-AS) ad libitum. All procedures in this study were according to the guidelines set by the Brazilian Animal Experimental College (COBEA).

\subsection{Ethics}

This study was approved by the Ethical Committee for the use of Experimental Animals of the Universidade Federal de Minas Gerais, Brazil (CETEA).

\subsection{Blood sample collection}

Peripheral blood $(5 \mathrm{ml})$ from the braquio cephalic vein was collected into EDTA containing tubes (final concentration of $1 \mathrm{mg} / \mathrm{ml}$ ). Hemogram was performed in each sample (Coulter MD18, USA). All samples were maintained at room temperature up to $12 \mathrm{~h}$ prior to processing.

\subsection{Immunophenotyping by flow cytometry}

\subsubsection{Monoclonal antibodies}

Unlabeled canine monoclonal antibodies anti-Thy1 (Rat-IgG2b-Clone YKIX337.217), anti-CD5 (RatIgG2a-Clone YKIX322.3), anti-CD4 (Rat-IgG2aClone YKIX302.9), anti-CD8 (Rat-IgG1-Clone YCATE55.9), anti-MHCII (Rat-IgG2b-Clone YKIX334.2), anti-CD45RA (Rat-IgG2b-Clone YKIX753.22.2) and anti-CD45RB (Rat-IgG2bClone YKIX716.13) were used in an indirect immunofluorescence procedure. Pooled rat normal serum diluted 1:6.000 was used as isotypic control for indirect immunoassay. FITC-labeled IgG sheep antirat polyclonal antibody was used as secondary antibody for indirect immunofluorescence. Nonspecific binding of second step reagent was blocked with pooled normal sheep serum in phosphate buffered saline (PBS) containing 10\% of fetal bovine serum-PBS/FBS (GIBCO, Grand Island, New York, USA).

FITC-labeled mouse anti-human-CD21 (MouseIgG1-Clone IOBla) and PE/Cy-5-conjugated mouse
anti-human-CD14 (Mouse-IgG2a-Clone TÜK4) mAbs were used in a direct immunofluorescence procedure.

In an attempt to identify the best dilution for each assay, mAbs were previously titered in a solution of PBS containing $1 \%$ bovine serum albumin and $0,1 \%$ sodium-azide (PBS-BSA).

Unlabeled mAbs and anti-CD14 mAbs used in this study were purchased from SEROTEC (Oxford, UK) and anti-CD21 from Immunotech Co. (Marselle, France).

\subsubsection{Preparation of pre-fix leukocyte suspension- erythrocyte-lysed and leukocyte-fixed whole blood samples}

A sample of $1 \mathrm{ml}$ of EDTA whole blood was transferred to a $15 \mathrm{ml}$ polypropylene tube, $\mathrm{V}$-shaped bottom (FALCON, Becton Dickinson, Moutain View, CA). Erythrocyte lysis and WBL pre-fixation were performed by slow addition of lysis solution until the total volume reached $13 \mathrm{ml}$ (FACS lysing solution (FLS), Becton Dickinson, Moutain View, CA) and immediately submitted to vigorous vortex agitation. The FLS is a hypotonic solution containing less than $50 \%$ diethylene glycol and less than $15 \%$ of formaldehyde able to preserve the WBL morphometric features and fix them prior immunophenotyping. The cellular suspension was incubated for $10 \mathrm{~min}$ at room temperature (RT) followed by centrifugation at $450 \times g$, for $10 \mathrm{~min}$, at RT. The supernatant was discarded and the pellet resuspended in $13 \mathrm{ml}$ of phosphate buffered saline (PBS $0.015 \mathrm{M}$, $\mathrm{pH}$ 7.2). The cell suspension was homogenized and submitted to an additional centrifugation at $450 \times g$, for $10 \mathrm{~min}$, at RT. The pellet was homogenized and resuspended in $10 \mathrm{ml}$ of $\mathrm{PBS} / \mathrm{fetal}$ bovine serum (FBS). After centrifugation at $450 \times g$, for $10 \mathrm{~min}$, at RT, the supernatant was aspirated, and the pellet resuspended in $500 \mu \mathrm{l}$ PBS/FBS. The number of events was previously adjusted for 1500 events/s/per tube. For quality control assessment, $60 \mu \mathrm{l}$ of pre-fix leukocyte suspension was added to $340 \mu$ of FACS FIX solution (10.0 g/l paraformaldehyde; $10.2 \mathrm{~g} / 1$ sodium cacodylate and $6.65 \mathrm{~g} / \mathrm{l}$ sodium chloride, $\mathrm{pH}$ 7.2) and run in the flow cytometer. The pre-fixed WBL suspension maintains their phenotypic profile stability after at least 1 month of long-term storage at $4{ }^{\circ} \mathrm{C}$. 


\subsubsection{Microplate assay for canine whole blood leukocytes phenotyping}

The protocol for immunophenotyping of fresh blood samples was carried out as follow: in $12 \mathrm{~mm} \times 75 \mathrm{~mm}$ polystyrene tubes, $30 \mu \mathrm{l}$ of fresh whole blood were incubated with $30 \mu l$ of previously diluted primary rat anti-canine cell surface markers at RT for 30 min in the dark. Following incubation, the cells were washed with $2 \mathrm{ml}$ of PBS and centrifuged at $800 \times g$ for $10 \mathrm{~min}$ at RT. The cells incubated with unlabeled primary antibody were reincubated at RT for $30 \mathrm{~min}$ in the dark, in the presence of $60 \mu \mathrm{l}$ of previously diluted FITC-conjugated sheep anti-rat IgG antibody. Labeled cells were fixed and the erythrocyte lysis performed by adding $2 \mathrm{ml}$ of lysis solution (FACS Lysing Solution, Becton Dickinson, Moutain View, CA) for $10 \mathrm{~min}$ in the dark at RT. Canine-WBL were washed twice with $2 \mathrm{ml}$ of PBS and centrifuged at $400 \times g$ for $10 \mathrm{~min}$ at RT. After the washing procedure, the labeled cells were fixed for 30 min with $300 \mu l$ of FACS FIX solution before analysis in the cytometer.

The immunofluorescence assay for pre-fix canineWBL was carried out as follows: in 96 wells, "U" bottom plate (LIMBRO Biomedicals, Inc. Aurora, Ohio), $30 \mu \mathrm{l}$ of canine-WBL suspensions were incubated at RT for $30 \mathrm{~min}$ in the dark, in the presence of $30 \mu \mathrm{l}$ of previously diluted anti-canine cell surface markers. Following incubation, the cells were washed with $140 \mu \mathrm{l}$ of PBS and centrifuged at $800 \times g$ for $10 \mathrm{~min}$ at RT. The cells incubated with unlabeled primary antibody were reincubated at RT for $30 \mathrm{~min}$ in the dark, in the presence of $60 \mu \mathrm{l}$ of previously diluted FITC-conjugated sheep anti-rat IgG antibody. After a second washing procedure with $200 \mu \mathrm{l}$ of PBS and centrifugation at $800 \times g$ for $10 \mathrm{~min}$ at RT, the labeled cells were fixed for $30 \mathrm{~min}$ with $200 \mu \mathrm{l}$ of FACS FIX solution before analysis in the cytometer.

Each assay included an internal control for nonspecific binding in which the cells were incubated with the same dilution of FITC-conjugated sheep anti-rat $\mathrm{IgG}$ antibody alone. The stained cells were stored at $4{ }^{\circ} \mathrm{C}$ up to $24 \mathrm{~h}$ before cytofluorometric analysis.

\subsubsection{Flow cytometry data storage and analysis}

Flow-cytometric measurements were performed on a FACScan instrument (Becton Dickinson,
Moutain View, CA) interfaced to an apple G3 Workstation. The Cell-Quest software package was used in both data acquisition and analysis. A total of 10.000 events were acquired for each preparation. Canine-WBL was identified on the basis of their specific forward (FSC) and side (SSC) light-scatter properties. Following FSC and SSC gain adjustments; the lymphocytes were detected based on their characteristic FSC versus SSC distribution. The lymphocytes were selected by gating on the FSC versus SSC graph. Fluorescence was evaluated based on the spectra of FITC and Cy5-PE on FL1 or FL3 single-histogram representation. The monocytes were analyzed by fluorescence intensity detection on single histograms directly on ungated leukocytes. For data analysis, a marker was set on the internal control for unspecific binding, in order to confine over $98 \%$ of the unlabeled cells. This marker was used in all data analysis for a given animal.

The results were expressed as percentage of positive cells within the selected gate, for cell surface markers presenting bimodal distribution. (Thy-1, CD5, CD4, CD8 and CD21), semi-quantitative analyses were performed to evaluate differential expression of cell surface markers presenting unimodal distribution (MHC-II, CD45RA and CD45RB). In these cases, the results were expressed as mean fluorescence intensity (MFI) on a log scale.

The data were also expressed in absolute counts, which allow the normalization of data from groups whose overall leukocytes counts may differ. The absolute counts for lymphocytes and monocytes were obtained as: (global leukocyte counts $\times$ percentage of lymphocytes or monocytes in hematoscopy)/100. Absolute counts for lymphocyte subsets were further calculated as: (lymphocyte absolute counts $\times$ percentage of fluorescent positive cells within lymphogate)/ 100.

\subsubsection{Statistical analysis}

Statistical analysis was performed using the Minitab 9.2 software package. One-way analysis of variance (ANOVA) was used for the comparative studies between groups. Student's $t$-test was used to identify significant differences between the group averages. The differences were considered significant when the probabilities of equality, $P$ values, were $\leq 0.05$. 


\section{Results}

\subsection{The use of pre-fix WBL in a microplate assay allows long-term sample storage}

In order to evaluate the effect of pre-fixation on the binding of anti-canine mAbs to the cells surface markers, we performed a study in which immunophenotyping of fresh whole blood samples were compared to a pre-fix preparations. Those experiments allowed for evaluate the molecular stability of canine cell surface markers after fixation with formaldehyde. Fig. 1 shows a typical profile of fresh (1A) and pre-fix WBL suspension (1B) regarding forward scattering (FSC-size) and side scattering (SSC-granularity) parameters (top dot plots). To further assess the phenotyping profile stability after long-term storage, pre-fix samples were stored at $4{ }^{\circ} \mathrm{C}$, for one month (Fig. 1C). The fresh and pre-fix samples used for comparison were derived from the same blood specimen from the same dog. Our data demonstrates that pre-fixation process (Fig. 1B) did not disturb the canine-WBL laser scattering properties, preserving FSC (size) versus SSC (internal complexity-granularity) profile when compared to fresh samples (Fig. 1A). Moreover, long-term storage (1 month) did not alter the referred parameters (Fig. 1C). Interestingly, we observed that except for CD14 marker on monocytes, structure of canine lymphocyte surface markers was preserved after fixation with formaldehyde, since labelling of pre-fix did not differ from those observed for fresh whole blood samples. Bottom histograms on Fig. 1 shows that CD14 was undetectable on pre-fix in comparison to fresh samples. However, the CD14 labelling was preserved after fixation with formaldehyde, once the labeling step was performed prior to fixation (Fig. 1C).

Semi-quantitative analyses of cell surface markers presenting unimodal distribution (MHC-II, CD45RA and CD45RB), were also stable for immunophenotyping after fixation, with no changes on fluorescence intensity distribution as determined by MFI on a log scale analysis (Fig. 2).

Using samples from different animals in parallel studies we certified that phenotypic stability after fixation was not an artifact (data not shown).
Based these data, we established the pre-fix condition as a new methodology for use in field studies.

\subsection{Decrease on lymphocyte and increase on monocyte counts are hematological hallmarks of canine aging}

Age-related changes on hematological features were investigated by automated hematological counting. In addition, conventional panoptic staining hematoscopy for leukocyte count assessment and differential analysis of leukocyte populations were also used.

Our data demonstrated that despite the fact that we did not observe any differences between sexes, hematological parameters showed a significant decrease on total lymphocyte and an increase of absolute monocyte counts in middle-aged-dogs when compared to young animals (Table 1). No significant differences in absolute counts between young and middle-aged dogs were observed for total leukocytes, granulocytes, neutrophils and eosinophils.

Taken together, these results show a typical lymphocytosis in young dogs, with lower counts as the animal ages. On the other hand, monocyte counts revealed a distinct profile of higher values in middleaged animals.

No significant differences were detected between males and females for all parameters analyzed.

\subsection{An increase on the percentage of $C D 5^{+} T$-cells, decrease on $C D 21^{+}$B-lymphocytes and unaltered $C D 4^{+} / C D 8^{+}$ratio are observed with aging}

In order to validate the microplate assay for canine flow cytometric assessment, we characterized canineWBL subsets in pre-fix preparations. A cross-sectional study was performed to evaluate the effect of aging on lymphocyte subpopulations in peripheral blood of normal dogs of both sexes (Table 2). Our data demonstrate a significant increase on the percentage of $\mathrm{CD5}^{+} \mathrm{T}$-cells in aged animals when compared to young dogs. In contrast, analysis of the B-cell population shows a striking decrease on the percentage of $\mathrm{CD} 21^{+}$cells in aged when compared to the young animals (Table 2). Together these findings demonstrate a progressive increase on $\mathrm{CD}^{+} / \mathrm{CD} 21^{+}$ cells with age. 

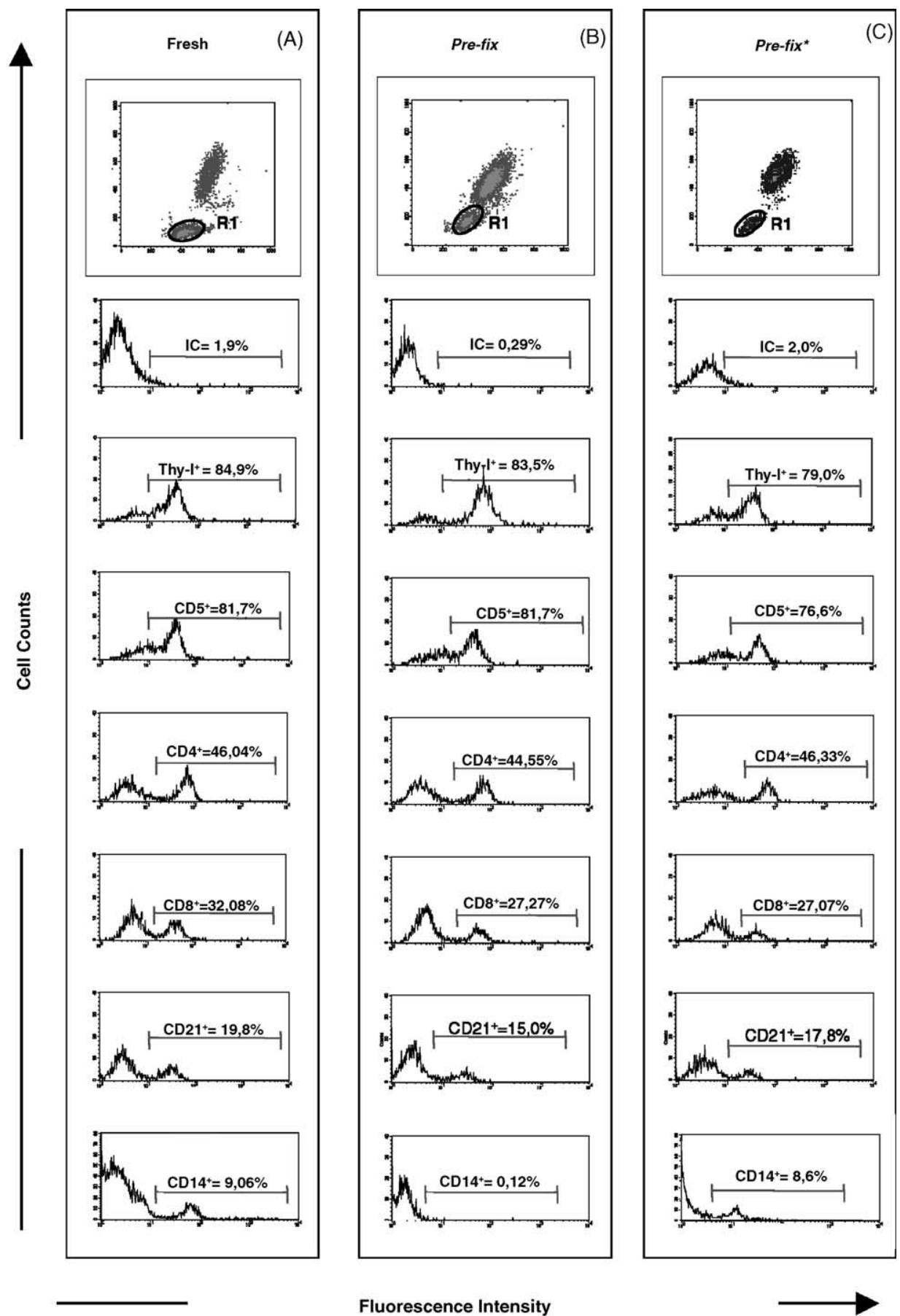

Fig. 1. Representative flow cytometry diagrams showing that molecular structure of canine lymphocyte surface markers is preserved after fixation of canine-WBL prior immunophenotyping. Fresh (A), pre-fix (B) and long-term stored pre-fix (C) were tested for their immunophenotyping features. As observed, despite fixation process canine-WBL assumes a characteristic homogeneous distribution that allows selective analysis of lymphocyte by creating a specific window R1 on laser scatter graphs—size (Forward Scatter—FSC) vs. granularity 

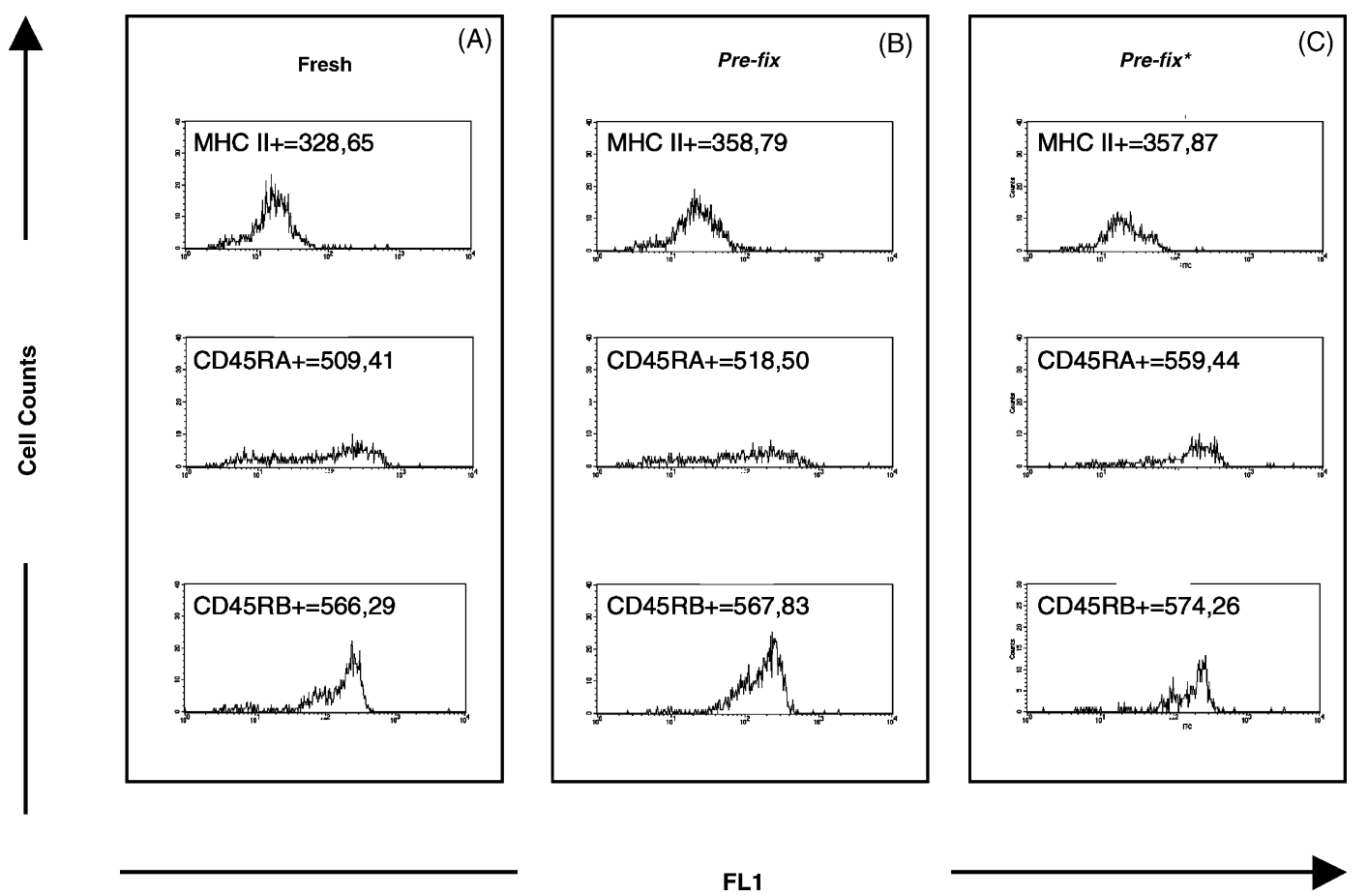

Fig. 2. Single color histograms of fluorescence intensity (FL1) representing labeled total lymphocyte population after staining with canine mAbs for MHC-II, CD45RA and CD45RB. Fresh (A), pre-fix (B) and long-term stored pre-fix (C) were tested for their immunophenotyping features. Histogram statistics were used for data analysis and the results were expressed as mean fluorescence intensity values on log scale for total lymphocytes. As observed, despite fixation process, no changes on curve shape of fluorescence intensity can be observed on single color histograms after staining with mAbs.

Analysis of non-T-non-B (NTNB) cells calculated as a resultant of (Thy- $1^{-}+\mathrm{CD} 21^{-}$) or $\left(\mathrm{CD}^{-}+\mathrm{CD} 21^{-}\right)$cells show a lower number of these cells in aged animals, with significant differences mainly on $\mathrm{CD}^{-} \mathrm{CD} 21^{-}$cells.

Analysis of the T-lymphocyte subpopulation is generally obtained after whole blood sample staining with a combination of anti-CD3, anti-CD4 and antiCD8 mAbs. Since pairs of mAbs labeled with distinct fluorochromes were not available at the time when this study was conduced, the samples were stained in a single color platform using anti-CD4 and anti-CD8 mAbs. Our data did not show any age-related significant differences on the percentages of T-cell subsets, or on $\mathrm{CD} 4^{+} / \mathrm{CD}^{+}$ratio.
No significant differences on the percentage of gated lymphocyte subsets were observed between males and females.

\subsection{Young dogs presented higher absolute counts of lymphocyte subsets in comparison with aged-animals}

To further investigate the influence of the aging process on canine peripheral blood lymphocyte subset pattern we focused our attention on the absolute counts of the major lymphocyte subsets. Therefore, to establish the reference values for normal $\operatorname{dogs}$ in different ages and both sexes we also calculated the absolute values of lymphocytes considering their

(side scatter-SSC). Moreover, no changes on neither curve shape of fluorescence intensity after staining with mAbs nor percentage of positive cells for THY-1, CD5, CD4, CD8 and CD21 can be observed on single color histograms. Bottom histograms illustrate that CD14 molecule was the only cell surface marker affected by the fixation process. However, the CD14 labelling was preserved after fixation, once the labeling step was performed prior to fixation (C). 
Table 1

Impact of age and sex on hematological features of healthy mongrel dogs

\begin{tabular}{|c|c|c|c|c|}
\hline \multirow[t]{3}{*}{ Hematological parameters } & \multicolumn{4}{|l|}{ Groups } \\
\hline & \multicolumn{2}{|l|}{ Young } & \multicolumn{2}{|l|}{ Middle-aged } \\
\hline & Male & Female & Male & Female \\
\hline Total leukocytes & $12.5 \pm 2.1$ & $13.1 \pm 1.9$ & $11.6 \pm 1.6$ & $13.5 \pm 2.9$ \\
\hline Granulocytes & $8.5 \pm 2.0$ & $9.0 \pm 1.6$ & $8.3 \pm 1.5$ & $10.6 \pm 2.8$ \\
\hline Neutrophils & $7.8 \pm 2.2$ & $8.0 \pm 2.3$ & $6.3 \pm 1.7$ & $9.0 \pm 2.2$ \\
\hline Eosinophils & $0.7 \pm 0.3$ & $1.0 \pm 0.4$ & $2.0 \pm 0.8$ & $1.6 \pm 0.3$ \\
\hline Lymphocytes & $3.5 \pm 0.6 \mathrm{c}$ & $3.7 \pm 0.7 \mathrm{~d}$ & $2.3 \pm 0.6 \mathrm{a}$ & $2.0 \pm 0.4 \mathrm{~b}$ \\
\hline \multicolumn{5}{|l|}{ Monocytes } \\
\hline Hematoscopy & $0.4 \pm 0.1 \mathrm{c}$ & $0.4 \pm 0.1 \mathrm{~d}$ & $1.1 \pm 0.5 \mathrm{a}$ & $0.7 \pm 0.3 \mathrm{~b}$ \\
\hline Flow cytometry & $0.9 \pm 0.3$ & $1.1 \pm 0.5$ & $1.0 \pm 0.5$ & $0.9 \pm 0.4$ \\
\hline
\end{tabular}

The results are expressed as mean cell counts-E03/ $\mathrm{mm}^{3} \pm$ standard deviation. The letters a, b, c and d represent statistically significant differences at $P \leq 0.05$ in comparison to young female, young male, middle-aged female and middle-aged male, respectively.

global count as described in Materials and Methods. Our data demonstrated that young dogs have higher absolute counts for T-lymphocyte subsets (Thy $1^{+}$, $\left.\mathrm{CD} 4^{+}, \mathrm{CD} 8^{+}\right)$, B-cells $\left(\mathrm{CD} 21^{+}\right)$and lower absolute counts for NTNB cells $\left(\mathrm{CD}^{-} \mathrm{CD} 21^{-}\right)$in peripheral blood when compared to aged-animals (Fig. 3). No significant difference was observed in the absolute counts of peripheral blood $\mathrm{CD}^{+}$T-lymphocytes among the groups analyzed (Fig. 3). Gender analysis did not show any statistically significant differences.

\subsection{Down regulation of $M H C-I I$ and CD45RA molecules on lymphocytes is an important immunological feature during aging}

Analysis of the expression of MCH-II and CD45 isoforms was performed by first gating on the

Table 2

Effect of age and sex on canine peripheral blood lymphocyte subsets profile of healthy mongrel dogs

\begin{tabular}{|c|c|c|c|c|}
\hline \multirow[t]{3}{*}{ Cell population } & \multicolumn{4}{|l|}{ Groups } \\
\hline & \multicolumn{2}{|l|}{ Young } & \multicolumn{2}{|l|}{ Middle-aged } \\
\hline & Male & Female & Male & Female \\
\hline \multicolumn{5}{|l|}{ T-lymphocytes } \\
\hline Thy-1 $1^{+}$ & $71.5 \pm 7.9$ & $73.1 \pm 10.5$ & $79.6 \pm 5.4$ & $76.9 \pm 7.8$ \\
\hline $\mathrm{CD}^{+}$ & $57.5 \pm 12.1 \mathrm{c}$ & $53.2 \pm 15.4 \mathrm{~d}$ & $74.6 \pm 6.1 \mathrm{a}$ & $73.8 \pm 6.8 \mathrm{~b}$ \\
\hline $\mathrm{CD}^{+}$ & $39.9 \pm 9.7$ & $40.1 \pm 10.9$ & $44.6 \pm 5.3$ & $46.4 \pm 8.8$ \\
\hline $\mathrm{CD}^{+}$ & $24.9 \pm 4.6$ & $24.6 \pm 6.0$ & $28.9 \pm 8.7$ & $26.7 \pm 8$ \\
\hline \multicolumn{5}{|l|}{ B-Lymphocytes } \\
\hline $\mathrm{CD} 21^{+}$ & $21.1 \pm 9.2 \mathrm{c}$ & $19.7 \pm 8.2 \mathrm{~d}$ & $10.1 \pm 3.7 \mathrm{a}$ & $14.9 \pm 7.1 \mathrm{~b}$ \\
\hline NTNB cells & & & & \\
\hline Thy $-1^{-} \mathrm{CD} 21^{-}$ & $13.7 \pm 6.6$ & $10.3 \pm 5.8 \mathrm{~d}$ & $11.0 \pm 0.1$ & $5.0 \pm 3.4 \mathrm{~b}$ \\
\hline $\mathrm{CD}^{-} \mathrm{CD} 21^{-}$ & $24.0 \pm 12.2 \mathrm{c}$ & $30.5 \pm 14.8 \mathrm{~d}$ & $15.05 \pm 4.2 \mathrm{a}$ & $10.7 \pm 5.0 \mathrm{~b}$ \\
\hline \multicolumn{5}{|l|}{ Lymphocyte ratios } \\
\hline Thy $-1^{+} / \mathrm{CD} 21^{+}$ & $3.6 \pm 1.2 \mathrm{c}$ & $4.3 \pm 2.0$ & $8.1 \pm 2.7 \mathrm{a}$ & $7.4 \pm 2.7$ \\
\hline $\mathrm{CD}^{+} / \mathrm{CD} 21^{+}$ & $2.6 \pm 1.1 \mathrm{c}$ & $2.9 \pm 1.3 \mathrm{~d}$ & $7.8 \pm 3.0 \mathrm{a}$ & $7.0 \pm 2.7 \mathrm{~b}$ \\
\hline $\mathrm{CD}^{+} / \mathrm{CD}^{+}{ }^{+}$ & $1.7 \pm 0.6$ & $1.7 \pm 0.5$ & $1.7 \pm 0.5$ & $1.9 \pm 0.8$ \\
\hline
\end{tabular}

The results are showed as the mean percentage \pm standard deviation of fluorescence positive cells into lymphogate. The percentage of NTNB cells was obtained as $100-\left(\%\right.$ Thy $\left.-1^{+}+\% \mathrm{CD} 21^{+}\right)$or $100-\left(\% \mathrm{CD}^{+}+\% \mathrm{CD} 21^{+}\right)$for each animal and data expressed as mean percentage \pm standard deviation. The letters a, b, c and d represent statistically significant differences at $P \leq 0.05$ in comparison to young female, young male, middle-aged female and middle-aged male, respectively. 

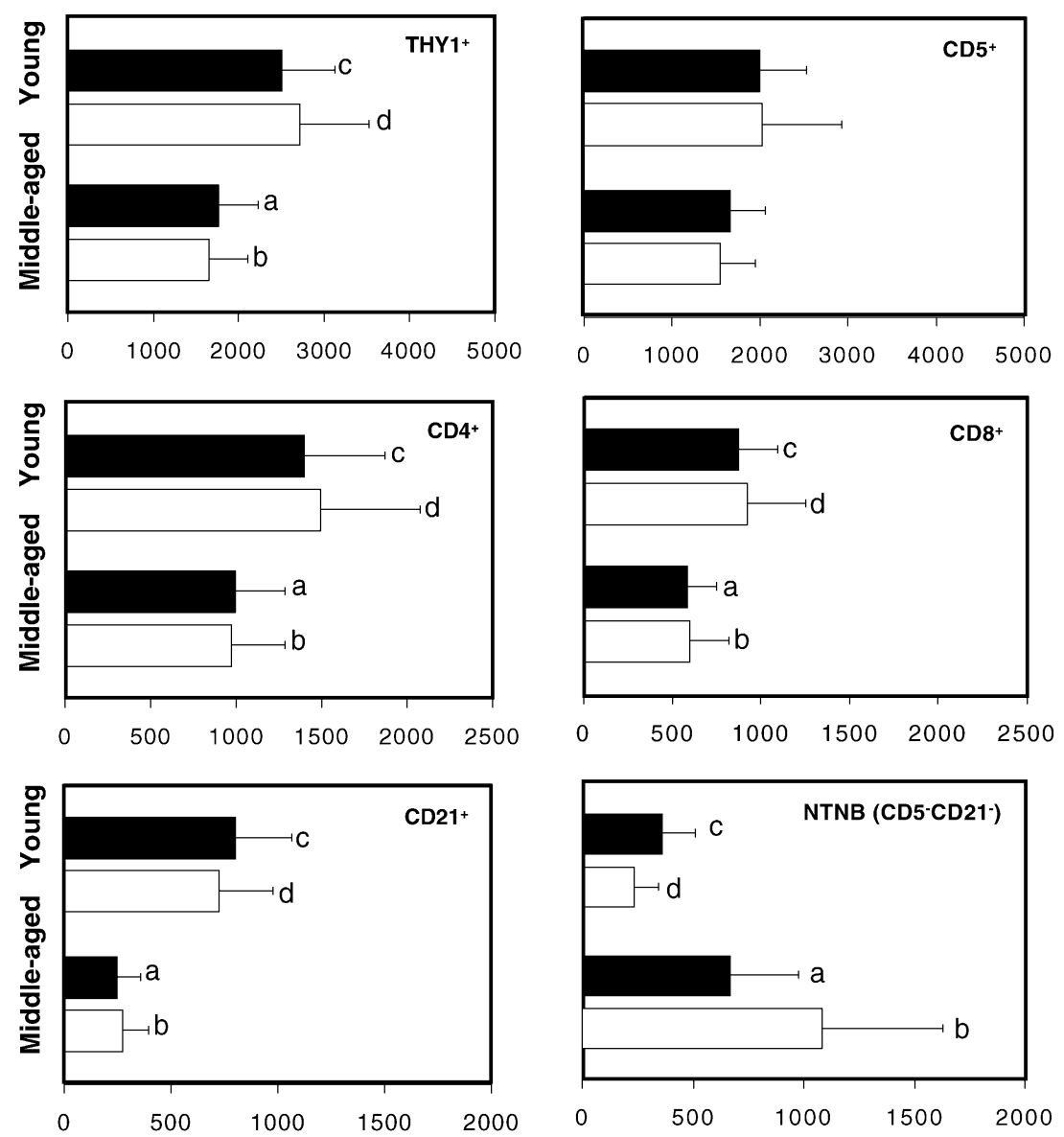

Fig. 3. Analysis of canine-WBL subsets in male ( $\square$ ) and female $(\square)$ healthy dogs with different age ranges. Phenotypic studies were performed using single color platform protocols, including anti-Thy-1, anti-CD5, anti-CD4 and anti-CD8 on indirect immunophenotyping assay and FITClabeled anti-CD21 on direct immunoassay within gated lymphocytes, as described in Section 2. Analysis of non-T-non-B ells (NTNB) was calculated as a resultant of $\left(\mathrm{CD}^{-}+\mathrm{CD} 21^{-}\right)$cells. The results are expressed as mean counts \pm standard deviation for lymphocytes subsets $/ \mathrm{mm}^{3}$ of blood. The letters a, b, $\mathrm{c}$ and d represent statistically significant differences at $P \leq 0.05$ in comparison to young female, young male, middleaged female and middle-aged male, respectively.

lymphocyte population followed by fluorescence intensity analysis on single color histograms as described in Section 2. The data was presented as mean fluorescence intensity on log scale for MHC-II and CD45 isoforms expression on gated lymphocytes (Fig. 4). Our results indicated a shift towards lower expression of MHC-II molecule by lymphocytes (MHC-II ${ }^{\text {low+ }}$ cells) in middle-aged in comparison to young animals. Evaluation of the CD45 isoforms also demonstrated a lower CD45RA expression on the surface of total lymphocytes from middle-aged dogs when compared to the young animals in both sexes.
No significant differences were detected in the CD45RB expression.

\section{Discussion}

Flow cytometry methods, have been intensively used as routine in human medicine and murine experimental biology, have been applied to different animal species including non-human primates as well as domestic animals such as cats and dogs (Williams, 1997; Byrne et al., 2000; Lafont et al., 2000). It 

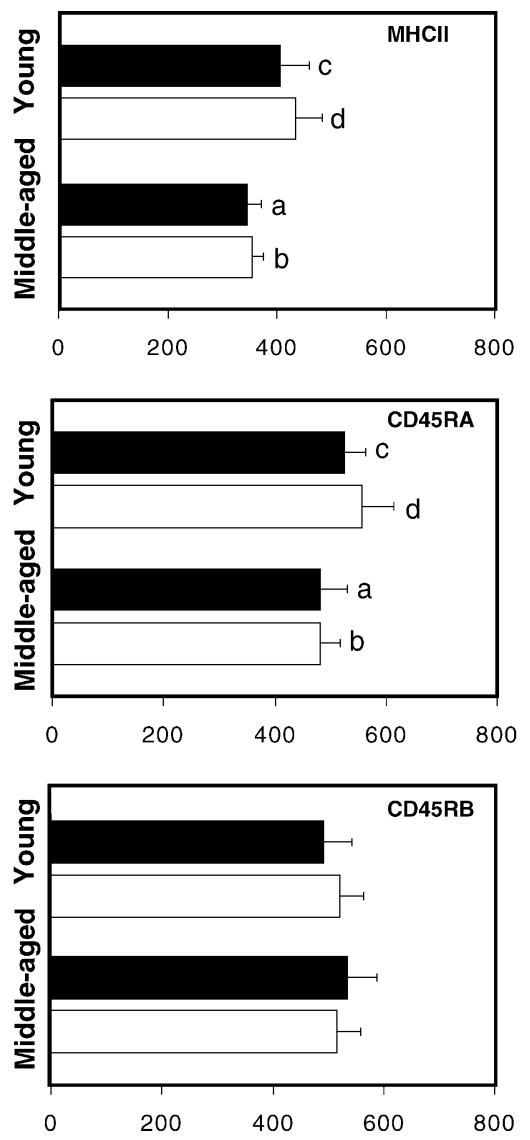

Fig. 4. Analysis of MHCII, CD45RA and CD45RO expression on total lymphocytes in male $(\square)$ and female $(\square)$ healthy dogs with different age ranges. Phenotypic studies were performed using single color platform protocols, including anti-MHCII, anti CD45RA and anti-CD45RB followed by incubation with FITClabeled anti-rat $\operatorname{IgG}$, as described in Section 2. The results are expressed as mean MFI \pm standard deviation. The letters $\mathrm{a}, \mathrm{b}, \mathrm{c}$ and $\mathrm{d}$ represent statistically significant differences at $P \leq 0.05$ in comparison to young female, young male, middle-aged female and middle-aged male, respectively.

provides a useful tool to rapidly quantify the relative percentage of cell subsets besides its important application in basic and clinical science. The effectiveness of its application, however, is dependent on standardization, especially in a clinical setting. For many years, application of flow cytometry to veterinary studies has been restricted by the lack of commercially available reagents (Cobbold and Metcalfe, 1994). Recently, the accessibility to monoclonal antibodies to canine cell surface markers has greatly stimulated research on the development of standar- dized protocols, which establishes the basis for comparisons between different methodologies (Byrne et al., 2000). Significant differences have been documented between phenotypic percentages for lymphocyte subsets measured by flow cytometry in healthy dogs. This fact can be explained, at least in part, by differences in immunophenotyping protocols, including sources and clones of monoclonal antibodies, methods for sample handling and data analysis. In addition, other variables such as breed, age, gender and nutrition status of the animals should be considered (Faldyna et al., 2001; Greeley et al., 2001; Heaton et al., 2002). This variation may be due also to the fact that there is no reference numbers for the various cell populations that could help on the standardization of the data. For these reasons, in the present investigation we focused our attention in providing researchers and clinicians with a standardized protocol for immunophenotyping of canineWBL. Further, we also demonstrate it is use evaluating age related phenotypic changes in normal animals.

A great advance of the present study is the perspective of fitting the whole blood immunophenotyping protocol to field applications. Despite studies demonstrating no differences in the experiments performed using PBMC isolated from freshly collected samples or those carried-out $2 \mathrm{~h}$ after collection or stored at $4{ }^{\circ} \mathrm{C}$ for $12-16 \mathrm{~h}$ before processing (Faldyna et al., 2001), no methodology has been published that focus on long-term sample storage. For this purpose, we have tested, in parallel studies, fresh, pre-fix and long-term stored pre-fix samples. Our observations demonstrate that phenotype and percentage of lymphocyte subsets were not adversely affected by sample pre-fixation and long-term storage, which are appropriate to the adequacy of the methodology to design field protocols. We have also observed, that CD14 molecule was the only one adversely affected by fixation. Similar data on other cell surface markers vulnerable to formaldehyde treatment have also been reported in humans (Macey et al., 1992). Since, CD14 immunophenotyping was affected by the fixation process, we suggest CD14 staining must be performed prior to fixation step.

Another gain besides the development of a microplate assay we applied the methodology to evaluate age-related phenotypic changes in canine peripheral blood leukocytes. The present study 
addresses the issue of (a) whether the hematological changes of senescence previously identified on PBMC could be adequately reproduced on whole blood immunophenotyping, and (b) whether specific immunological parameters are particularly informative to monitor canine aging process.

As clinical applications of flow cytometry has became a routine practice (Chabanne et al., 2000), the establishment of ranges for phenotype percentages of healthy animals is important as reference data to monitor alterations associated with disease. Herein, percentual analysis of lymphocyte subsets were examined in young and middle-aged mongrel dogs from 4 month to 6 years of age. Analysis of phenotype percentages of gated lymphocytes revealed a significant age-related increase of $\mathrm{CD}^{+} \mathrm{T}$-cells and a decline of $\mathrm{CD} 21^{+} \mathrm{B}$-cells that led to an increased T/B cell ratio. Percentages of T- and B-lymphocyte have been reported in different studies using PBMC phenotyping. In agreement with our findings, there is a major consensus of increased percentages of $\mathrm{T}$ and decreased percentage of B-cells in middle-aged dogs in comparison to young ones (Davila et al., 1992; Greeley et al., 1996; Faldyna et al., 2001; Toman et al., 2002). Significant age-related changes in percentage of $\mathrm{CD}^{+}$and $\mathrm{CD}^{+}$T-cell subsets have not been documented on the present study. In contrast with our data, the major discrepancy identified by other authors is an increased percentage of $\mathrm{CD}^{+} \mathrm{T}$-cells in middleaged dogs in comparison to younger animals. Gender differences were observed only on the percentages of T-cells, with females having higher values (Davila et al., 1992; Greeley et al., 1996; Faldyna et al., 2001). These data in contrast with those presented on a longitudinal study which examined $\mathrm{CD} 4^{+} / \mathrm{CD}^{+}$ratios did not reveal any additional age or gender differences (Greeley et al., 2001).

While the number and distribution of blood leukocyte of healthy individuals do not reflect specific immune responsiveness, they are useful indicators of the adequacy of the immune machinery. There is no consensus in the literature regarding age-related changes in number of leukocytes. In the present short-term cross-sectional aging canine study, monitoring of leukocyte counts from 4 month to 6 years of age revealed minimal change. Absolute counts of total leukocytes, granulocytes (both neutrophils and eosinophils) remained relatively stable, while absolute number of lymphocytes exhibited a significant decline with age in both genders. Analysis of monocytes showed an age-related increase on hematoscopy, not detected by flow cytometry. The hematological picture observed in this study is similar to the ranges previously described in the canine literature (Greeley et al., 2001), and are comparable in those observed in human and other species (ComansBitter et al., 1997; Shahabuddin et al., 1998; De Vries et al., 2000; Felsburg, 2002) where there is an increase in lymphocyte populations after birth and a decrease with the advancing of age. In young animals the lymphocytosis is similar to humans as well as in other species (Panaro et al., 1991).

The estimation of the percentage of NTNB cells (NK-like cells) was performed by an approximation of the sum of $\mathrm{T}$ - and B-cells to the total gated lymphocytes, since there are no clear markers for canine NK cells (Vitale et al., 1992). Based on this approach we identified an important age-related decrease on NTNB cells, mainly when analyzing $\mathrm{CD} 5^{-} \mathrm{CD} 21^{-}$cells. This decrease may translate into an overall decrease in functional killing capacity, several studies suggest that the number of killer cells become compromised with age (Davila et al., 1992; Vitale et al., 1992; Mariane et al., 1998; Borrego et al., 1999; Greeley et al., 2001). In contrast others reported an age-related increase in NK-activity (Strasser et al., 2000). There is no clear explanation for the differences observed in this study.

It has been proposed that absolute counts should be routinely performed by clinical laboratories to aid on the evaluation of individual cases (Byrne et al., 2000). In this study we determined the absolute counts of lymphocyte subpopulations and observed that the age-related lymphopenia led to a significant decline on all lymphocyte subsets evaluated, including $\mathrm{T}\left(\right.$ Thy $-1^{+}, \mathrm{CD}^{+}{ }^{+}$and $\left.\mathrm{CD} 8^{+}\right), \mathrm{B}\left(\mathrm{CD} 21^{+}\right)$and NTNB $\left(\mathrm{CD} 5^{-} \mathrm{CD} 21^{-}\right)$cells. In a longitudinal study in Labrador Retrievers a significant decline was demonstrated in the number of T-cells, $\mathrm{CD} 4^{+}$and $\mathrm{CD} 8^{+}$as a function of age (Greeley et al., 2001). Whether these markers of chronological age will be useful as indicators of immunological changes with depend on their correlation with the immunological status of the animal. At present, correlates of immunological measures taken in early or middle-aged with ultimate life span have been established only in mice and 
human (Ferguson et al., 1995; Heller et al., 1998; Wikby et al., 1998).

A major lacuna in the panel of antibodies available for canine immunophenotyping is on reagents to study activation and memory phenotypes, such as IL2-R. Several groups have reported potential lymphocyte activation markers at the CLAW meeting (Cobbold and Metcalfe, 1994), including differential expression of MHC-II molecules as well as CD45 isoforms. While there has been some debate about functional significance of differential expression of these molecules, some studies have suggested that these molecules are powerful candidates as activation markers for canine lymphocytes. Both T- and Blymphocytes express canine MHC-II molecules in contrast with man and rodent where only activated Tcells express the molecule. MHC-II molecules was evaluated on canine lymphocytes from birth to 8 months of age and observed an increase on the expression of this molecule by canine T-cells over time (Holmes and Lunn, 1994). These authors suggested that increased expression of the MHC-II might relate to exposure of the growing animal to environmental stimuli. CD45 antigen is expressed on all cells of hematopoietic origin, apart from erythrocytes, and is known as the leukocyte common antigen. It appears that naïve T-cells express CD45 with the exon unspliced while activated and memory cells express fully spliced CD45RO isoforms. One study in canine mycosis demonstrated the lack of CD45RA expression, interpreted as indicative of a memory phenotype (Moore et al., 1994).

Since both, MHC-II molecules and CD45 isoforms are expressed on all lymphocytes and therefore, show a unimodal fluorescence distribution, semi-quantitative analyses were performed to evaluate differential expression of these cell surface markers. In these cases, the results were expressed as mean fluorescence intensity on log scale. Our observations also points out to an age-related down-regulation of the MHC-II on circulating lymphocytes. We hypothesize that this phenomenon may extend to immunoregulatory or tolerance mechanisms acquired as a function of the continuous exposure to environmental stimuli. High levels of MHC-II expression in younger animals may reflect early immune system stimulation of young pups (Holmes and Lunn, 1994). Future studies are necessary to clarify the relationship between down- regulation of MHC-II expression and different pathways of canine T-cell responsiveness triggered by immunoregulatory events. To the monitoring of the expression of canine MHC-II molecule by flow cytometry will be helpful for further characterization of the canine immune system. The findings of the present study support our prediction that immunological memory that occurs in the individual over time do reflect the changes on lymphocyte phenotype characteristics, since middle-aged animals presented lower CD45RA expression, compatible with expected memory phenotype of these animals due to higher exposure to immunological stimuli over life.

In conclusion, our observations on age-related changes lymphocyte subsets distribution in mongrel dogs highlights the importance of choosing the correct immunological markers for the evaluation of agerelated effects which are appropriated to monitor oncohematological, auto-immune and infectious diseases. Our microplate pre-fix flow cytometry methodology associated with conventional hemogram of the canine peripheral whole blood provides a powerful application for accurate determination of relative phenotype percentages and absolute number of leukocytes and lymphocyte subsets. The results of this study provide important reference values for future investigations involving peripheral whole blood from mongrel dogs, and middle-aged versus young dogs. This study also represents and important first step in obtaining meaningful senescence-related changes in whole blood parameters previously identified on PBMC.

\section{Acknowledgements}

This work was supported by the $\mathrm{CNPq} / \mathrm{BR} /$ grant: 521124/98-0 and FAPEMIG/BR/grant: CBS 2222197.

\section{References}

Borrego, F., Alonso, M.C., Galiani, M.D., Carracedo, J., Ramirez, R., Ostos, B., Pena, J., Solana, R., 1999. NK phenotypic markers and IL-12 reponse in NK cells from elderly people. Exp. Gerontol. 34, 253-265.

Byrne, K.M., Reinhart, G.A., Hayek, M.G., 2000. Standardized flow cytometry gating in veterinary medicine. Meth. Cell Sci. 22, 191-198. 
Chabanne, L., Bonnefont, C., Bernaud, J., Rigal, D., 2000. Clinical applications of flow cytometry and cell immunophenotyping to companion animals (dog and cat). Meth. Cell Sci. 22, 199-207.

Cobbold, S., Metcalfe, S., 1994. Monoclonal antibodies that define canine homologues of human CD antigens: summary of the first international canine leukocyte antigen workshop (CLAW). Tiss. Antigens 43, 137-154.

Comans-Bitter, W.M., de Groot, R., van den Beemd, R., Neijens, H.J., Hop, W.C., Groeneveld, K., Hooijkaas, H., van Dongen, J.J., 1997. Immunophenotyping of blood lymphocytes in childhood, reference values for lymphocyte subpopulations. J. Pediatr. 130, 388-393.

Davila, D.R., Guilmette, R.A., Bice, D.E., Muggenburg, B.A., Swafford, D.S., Haley, P.J., 1992. Long-term consequences of ${ }^{239} \mathrm{PuO}_{2}$ exposure in dogs: persistent T-lymphocyte dysfunction. Int. J. Radiat. Biol. 61, 123-133.

De Vries, E., de Bruin-Versteeg, S., Comans-Bitter, W.M., de Groot, R., Hop, W.C., Boerma, G.J., Lotgering, F.K., van Dongen, J.J., 2000. Longitudinal survey of lymphocyte subpopulations in the first year of life. Pediatr. Res. 47, 528-537.

Faldyna, M., Leva, L., Knotigova, P., Toman, M., 2001. Lymphocyte subsets in peripheral blood of dogs-a flow cytometric study. Vet. Immunol. Immunopathol. 82, 23-37.

Felsburg, P.J., 2002. Overview of immune system development in the dog: comparison with humans. Hum. Exp. Toxicol. 21, 487492.

Ferguson, F.G., Wikby, A., Maxson, P., Olsson, J., Johansson, B., 1995. Immune parameters in a longitudinal study of a very old population of Swedish people: a comparison between survivors and nonsurvivors. J. Gerontol. A. Biol. Sci. Med. Sci. 50, 378382.

Greeley, E.H., Ballam, J.M., Harrison, J.M., Kealy, R.D., Lawler, D.F., Segre, M., 2001. The influence of age and gender on the immune system: a longitudinal study in Labrador Retriever dogs. Vet. Immunol. Immunopathol. 82, 57-71.

Greeley, E.H., Kealy, R.D., Ballam, J.M., Lawler, D.F., Segre, M., 1996. The influence of age on the canine immune system. Vet. Immunol. Immunopathol. 55, 1-10.

Heaton, P.R., Blount, D.G., Devlin, P., Koelsch, S., Mann, S.J., Smith, B.H., Stevenson, J., Harper, E.J., 2002. Assessing agerelated changes in peripheral blood leukocyte phenotypes in Labrador retriever dogs using flow cytometry. J. Nutr. 132, 1655S-1657S.

Heller, D.A., Ahern, F.M., Stout, J.T., McClearn, G.E., 1998. Mortality and biomarkers of aging in heterogeneous stock (HS) mice. J. Gerontol. A. Biol. Sci. Med. Sci. 53, 217-230.

Holmes, M.A., Lunn, D.P., 1994. Variantion of MCH II expression on canine lymphocytes with age. Tiss. Antigens 43, 179-183.

Jaroszeski, M.J., Radcliff, G., 1999. Fundamentals of flow cytometry. Mol. Biotechnol. 11, 37-53.
Lafont, B.A.P., Gloeckler, L., D’Hautcourt, J.L., Gut, J.P., Aubertin, A.M., 2000. One-round determination of seven leukocyte subsets in rhesus macaque blood by flow cytometry. Cytometry 41 , 193-202.

Macey, M.G., Jiang, X.P., Veys, P., Mc Carthy, D., 1992. Newland AC. Expression of functional antigens on neutrophils. Effects of preparation. J. Immunol. Meth. 149, 37-42.

Mariane, E., Mariane, A.R., Meneghetti, A., Tarozzi, A., Cocco, L., Facchini, A., 1998. Age-dependent decreases of NK cell phosphoinositide turnover during spontaneous but not Fc-mediated cytolytic acitivity. Int. Immunol. 10, 981-989.

Moore, P.F., Olivery, T., Naydan, D., 1994. Canine cutaneous epitheliotropic lymphoma (mycosis fungoides) is a proliferative disorder of $\mathrm{CD}^{+} \mathrm{T}$ cells. A. J. Pathol. 144, 421-429.

Otani, I., Niwa, T., Tajima, N., Ishikawa, A., Watanabe, T., Tsumagari, S., Takeishi, M., Kanayama, K., 2002. CD56 is expressed exclusively on $\mathrm{CD}^{+} \mathrm{T}$ lymphocyte in canine peripheral blood. J. Vet. Sci. 64, 441-444.

Panaro, A., Amati, A., di Loreto, M., Felle, R., Ferrante, M., Papadia, A.M., Porfido, N., Gambatesa, V., Dell'Osso, A., Lucivero, G., 1991. Lymphocyte subpopulations in pediatric age. Definition of reference values by flow cytometry. Allergol. Immunopathol. (Madr.) 19, 109-112.

Pedersen, N.C., Pool, R., 1978. Canine joint disease. Vet. Clin. North Am. 8, 465-493.

Pinelli, E., 1997. Cytokines in canine visceral Leishmaniasis. In: Virgil, E.C.J., Schijns, V.E.C.J., Horzinek, M.C. (Eds.), Cytokines in Veterinary Medicine. Cab International, United Kingdom, pp. 217-224.

Shahabuddin, S., Al-Ayed, I., Gad El-Rab, M.O., Qureshi, M.I., 1998. Age-related changes in blood lymphocyte subsets of Saudi Arabian healthy children. Clin. Diag. Lab. Immunol. 5, 632635.

Strasser, A., Teltscher, A., May, B., Sanders, C., Niederüller, H., 2000. Age-associated changes in the immune system of German Shephard dogs. J. Vet. Med. A 47, 181-192.

Toman, M., Faldyna, M., Knotigova, P., Pokorova, D., Sinkora, J., 2002. Postnatal development of leukocyte subset composition and activity in dogs. Vet. Immunol. Immunopathol. 87, 321-326.

Vitale, M., Zamai, L., Néri, L.M., Galanzi, A., Facchini, A., Rana, R., Cataldi, A., Papa, S., 1992. The impairment of natural killer function in the healthy aged is due to a postbinding deficient mechanism. Cell Immunol. 145, 1-10.

Wikby, A., Maxson, P., Olsson, J., Johansson, B., Ferguson, F.G., 1998. Changes in CD8 and CD4 lymphocyte subsets, T-cell proliferation responses, and non-survival in the very old: the Swedish longitudinal OCTO-immune study. Mech. Ageing Dev. 102, 187-198.

Williams, D.L., 1997. Studies of canine leukocyte antigens: A significant advance in canine immunology. The Vet. J. 153, 31-39. 